\title{
Effect of aspect ratio on vortex distribution and heat transfer in rotating Rayleigh-Bénard convection
}

Citation for published version (APA):

Stevens, R. J. A. M., Overkamp, J. V., Lohse, D., \& Clercx, H. J. H. (2011). Effect of aspect ratio on vortex distribution and heat transfer in rotating Rayleigh-Bénard convection. Physical Review E - Statistical, Nonlinear, and Soft Matter Physics, 84(5), 056313-1/10. [056313]. https://doi.org/10.1103/PhysRevE.84.056313

DOI:

10.1103/PhysRevE.84.056313

Document status and date:

Published: 01/01/2011

\section{Document Version:}

Publisher's PDF, also known as Version of Record (includes final page, issue and volume numbers)

\section{Please check the document version of this publication:}

- A submitted manuscript is the version of the article upon submission and before peer-review. There can be important differences between the submitted version and the official published version of record. People interested in the research are advised to contact the author for the final version of the publication, or visit the $\mathrm{DOI}$ to the publisher's website.

- The final author version and the galley proof are versions of the publication after peer review.

- The final published version features the final layout of the paper including the volume, issue and page numbers.

Link to publication

\section{General rights}

Copyright and moral rights for the publications made accessible in the public portal are retained by the authors and/or other copyright owners and it is a condition of accessing publications that users recognise and abide by the legal requirements associated with these rights.

- Users may download and print one copy of any publication from the public portal for the purpose of private study or research.

- You may not further distribute the material or use it for any profit-making activity or commercial gain

- You may freely distribute the URL identifying the publication in the public portal.

If the publication is distributed under the terms of Article 25fa of the Dutch Copyright Act, indicated by the "Taverne" license above, please follow below link for the End User Agreement:

www.tue.nl/taverne

Take down policy

If you believe that this document breaches copyright please contact us at:

openaccess@tue.nl

providing details and we will investigate your claim. 


\title{
Effect of aspect ratio on vortex distribution and heat transfer in rotating Rayleigh-Bénard convection
}

\author{
Richard J. A. M. Stevens, ${ }^{1}$ Jim Overkamp, ${ }^{2}$ Detlef Lohse, ${ }^{1}$ and Herman J. H. Clercx ${ }^{2,3}$ \\ ${ }^{1}$ Department of Science and Technology and J.M. Burgers Center for Fluid Dynamics, University of Twente, P.O. Box 217, \\ 7500 AE Enschede, The Netherlands \\ ${ }^{2}$ Department of Physics and J.M. Burgers Center for Fluid Dynamics, Eindhoven University of Technology, P.O. Box 513, \\ 5600 MB Eindhoven, The Netherlands \\ ${ }^{3}$ Department of Applied Mathematics, University of Twente, P.O. Box 217, 7500 AE Enschede, The Netherlands
}

(Received 23 May 2011; published 18 November 2011)

\begin{abstract}
Numerical and experimental data for the heat transfer as a function of the Rossby number Ro in turbulent rotating Rayleigh-Bénard convection are presented for the Prandtl number $\operatorname{Pr}=4.38$ and the Rayleigh number $\mathrm{Ra}=2.91 \times 10^{8}$ up to $\mathrm{Ra}=4.52 \times 10^{9}$. The aspect ratio $\Gamma \equiv D / L$, where $L$ is the height and $D$ the diameter of the cylindrical sample, is varied between $\Gamma=0.5$ and 2.0. Without rotation, where the aspect ratio influences the global large-scale circulation, we see a small-aspect-ratio dependence in the Nusselt number for $\mathrm{Ra}=2.91 \times 10^{8}$. However, for stronger rotation, i.e., $1 / \mathrm{Ro} \gg 1 / \mathrm{Ro}_{c}$, the heat transport becomes independent of the aspect ratio. We interpret this finding as follows: In the rotating regime the heat is mainly transported by vertically aligned vortices. Since the vertically aligned vortices are local, the aspect ratio has a negligible effect on the heat transport in the rotating regime. Indeed, a detailed analysis of vortex statistics shows that the fraction of the horizontal area that is covered by vortices is independent of the aspect ratio when $1 / \mathrm{Ro} \gg 1 / \mathrm{Ro}_{c}$. In agreement with the results of Weiss et al. [Phys. Rev. Lett. 105, 224501 (2010)], we find a vortex-depleted area close to the sidewall. Here we show that there is also an area with enhanced vortex concentration next to the vortex-depleted edge region and that the absolute widths of both regions are independent of the aspect ratio.
\end{abstract}

DOI: 10.1103/PhysRevE.84.056313

PACS number(s): 47.27.te, 47.32.Ef, 47.27.ek

\section{INTRODUCTION}

The classical system to study turbulent thermal convection in confined space is the Rayleigh-Bénard (RB) system, i.e., fluid between two parallel plates heated from below and cooled from above $[1,2]$. For given aspect ratio $\Gamma \equiv D / L$ ( $D$ is the sample diameter and $L$ its height) and given geometry, its dynamics are determined by the Rayleigh number $\mathrm{Ra}=\beta g \Delta L^{3} / \kappa \nu$ and the Prandtl number $\operatorname{Pr}=\nu / \kappa$. Here $\beta$ is the thermal expansion coefficient, $g$ is the gravitational acceleration, $\Delta$ is the temperature difference between the plates, and $v$ and $\kappa$ are the kinematic and thermal diffusivity, respectively. The case where the RB system is rotated around a vertical axis at an angular speed $\Omega$, i.e., rotating Rayleigh-Bénard (RRB) convection, is interesting for industrial applications and problems in geology, oceanography, climatology, and astronomy. The rotation rate of the system is nondimensionalized in the form of the Rossby number Ro $=\sqrt{\beta g \Delta / L} / 2 \Omega$, which represents the ratio between buoyancy and the Coriolis force.

It is well know that the boundary layer behavior plays an important roll in the heat transfer properties of a RB system [1,3-6]. However, several studies have shown that also the general flow structure in the system can influence the overall heat transfer. For nonrotating RB convection, Sun, $\mathrm{Xi}$, and $\mathrm{Xia}$ [7] demonstrated that the global transport in a turbulent flow system can depend on internal flow structures. Later $\mathrm{Xi}$ and $\mathrm{Xia}$ [8] quantified the difference in Nusselt number between the single-roll state (SRS) and double-roll state (DRS). Recently, Weiss and Ahlers [9] found that the difference in the heat transport between the SRS and DRS decreases with increasing Ra. Subsequently, van der Poel et al. [10] found that in two-dimensional RB convection a different flow structure can change the heat transfer up to $30 \%$.
An overview on the importance of the internal flow structure on the heat transfer is given by Xia [11]. The mechanism of the effect of the bulk flow on the heat transfer is as follows: More efficient bulk flow transports hot (cold) plumes to the top (bottom), thus creating a larger mean temperature gradient there and thus a larger $\mathrm{Nu}$. In this paper we will see that also in rotating RB convection the internal flow structures influence the overall heat transport in the system [12-18].

It has been shown by several authors that three different regimes can be identified in RRB convection [12-18]. As a function of increasing rotation rate, one first finds a regime without any heat transport enhancement at all in which the large-scale circulation (LSC) is still present (regime I). Zhong and Ahlers [17] showed that, though the Nusselt number is unchanged in this regime, various properties of the LSC do change with increasing rotation in this regime. Here we mention the increase in the temperature amplitude of the LSC, the LSC precession (also observed by Hart et al. [19] and Kunnen et al. [20]), the decrease of the temperature gradient along the sidewall, and the increased frequency of cessations. The start of regime II (moderate rotation) is indicated by the onset of heat transport enhancement due to Ekman pumping as is discussed by Zhong et al. [13] and Weiss et al. [15,21]. When the rotation rate is increased in regime II the heat transfer increases further until one arrives at regime III (strong rotation), where the heat transfer starts to decrease. This decrease of the heat transfer in regime III is due to the suppression of the vertical velocity fluctuations $[13,17,22]$.

Several experimental $[13,14,17,23-26]$ and numerical [13,14,16,20,22,26-31] studies on RRB convection have shown that in regime II the heat transport with respect to the nonrotating case increases due to rotation. A detailed overview 

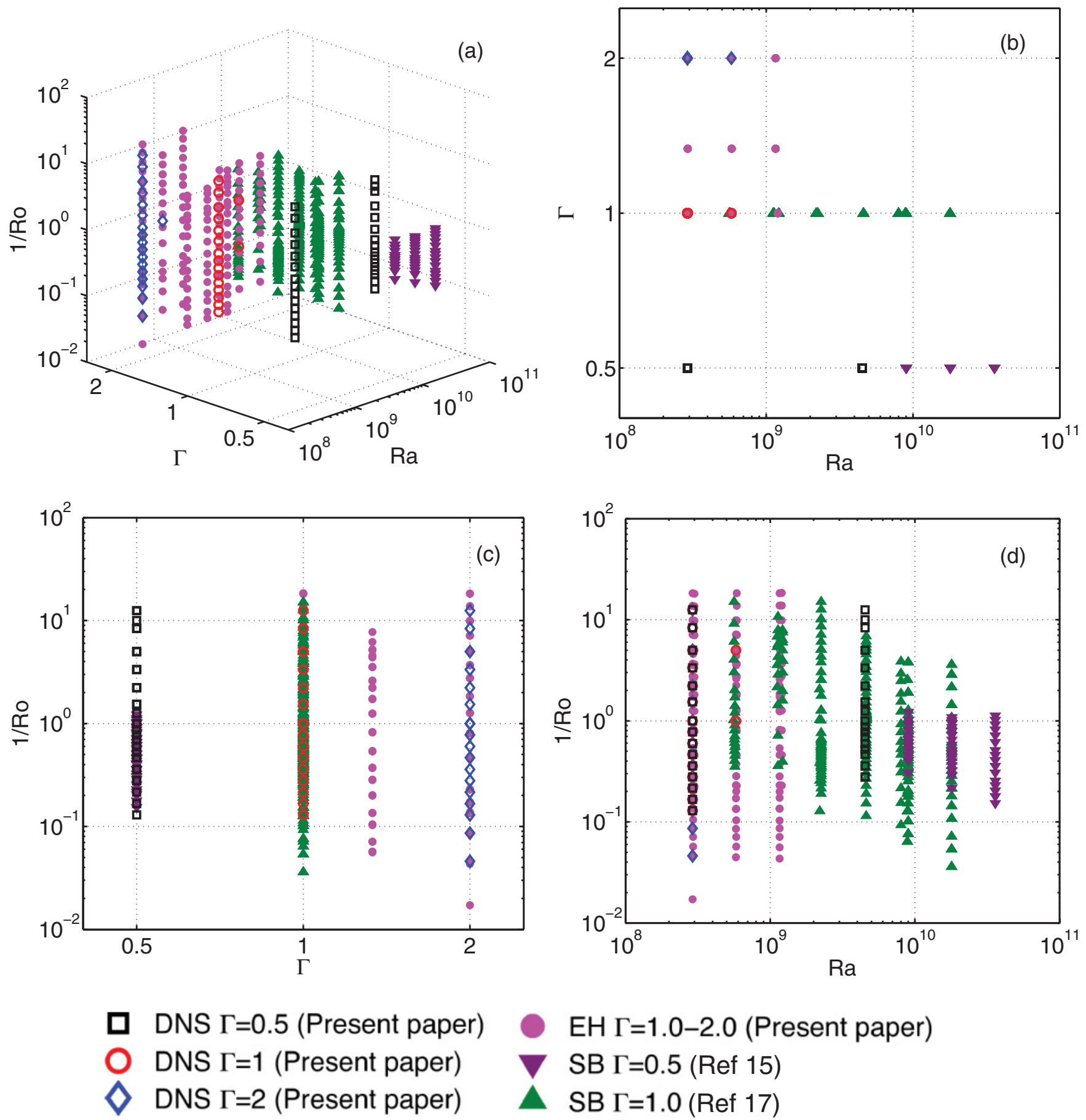

FIG. 1. (Color online) Phase diagram in Ra-Ro- $\Gamma$ space for RRB convection with $\operatorname{Pr}=4.38$. The data points indicate where Nu has been experimentally measured or numerically calculated in a cylindrical sample with no-slip boundary conditions. The DNS data and the Eindhoven (EH) experiments are from this study. The Santa Barbara (SB) experimental results are from Weiss et al. [15] and Zhong and Ahlers [17] for $\Gamma=0.5$ and 1.0, respectively. The EH experimental data focus on $\Gamma>1$, but for one case we also give the result for $\Gamma=1$ for benchmarking with the earlier SB data. (a) Three-dimensional view on the phase space [38], (b) projection on $\Gamma$-Ra phase space, (c) projection on 1/Ro- $\Gamma$ phase space, and (d) projection on 1/Ro-Ra phase space. We also refer to Fig. 1 of Stevens et al. [32] for a full representation of the Ra-Pr-Ro phase space of RRB convection.

of the parameter ranges covered in the different experiments can be found in the Ra-Pr-Ro phase diagram shown in Fig. 1 of Stevens et al. [32] and the Ra-Ro- $\Gamma$ phase diagram shown in Fig. 1. The heat transport enhancement in regime II is caused by Ekman pumping [13,17,20,23,26,27,33,34].
Namely, whenever a plume is formed, the converging radial fluid motion at the base of the plume [in the Ekman boundary layer (BL)] starts to swirl cyclonically, resulting in the formation of vertical vortex tubes. The rising plume induces stretching of the vertical vortex tube and hence additional 
vorticity is created. This leads to enhanced suction of hot fluid out of the local Ekman layer and thus increased heat transport. Corresponding phenomena occur at the upper boundary.

Zhong et al. [13] and Stevens et al. [14,22] used results from experiments and direct numerical simulations (DNSs) in a $\Gamma=1$ sample to study the influence of $R a$ and Pr on the effect of Ekman pumping. It was found that at fixed Ro the effect of Ekman pumping is largest and thus the observed heat transport enhancement with respect to the nonrotating case highest, at an intermediate Prandtl number. At lower Pr the effect of Ekman pumping is reduced as more hot fluid that enters the vortices at the base spreads out in the middle of the sample due to the large thermal diffusivity of the fluid. At higher Pr the thermal $\mathrm{BL}$ becomes thinner with respect to the kinetic BL, where the base of the vortices is formed, and hence the temperature of the fluid that enters the vortices becomes lower. In addition, it was found that the effect of Ekman pumping is reduced for increasing Ra. This is because the turbulent viscosity increases with increasing $\mathrm{Ra}$, which means that more heat spreads out in the middle of the sample. This explains why for the high Ra number of Niemela et al. [35] no heat transfer enhancement was found in the rotating case. From this we can conclude that the internal flow structures influence the heat transfer in rotating $\mathrm{RB}$ convection as is also the case for nonrotating $\mathrm{RB}$ convection [7-10].

Recently, Weiss et al. $[15,21]$ showed that the rotation rate at which the onset of heat transport enhancement sets in $\left(1 / \mathrm{Ro}_{c}\right)$ increases with decreasing aspect ratio due to finite-size effects. This means that the aspect ratio is an important parameter in RRB convection. We report in this paper on a systematic study of the influence of the aspect ratio on heat transfer (enhancement) for moderate and strong rotation rates, i.e., for $1 / \operatorname{Ro} \gg 1 / \mathrm{Ro}_{c}$. We start this paper with a description of the experimental and numerical procedures that have been followed. Where the experimental and simulation results overlap, excellent agreement is found. Based on the numerical data, we will show that for $1 / \mathrm{Ro} \gg 1 / \mathrm{Ro}_{c}$ the Nusselt number is independent of the aspect ratio, while there are some visible differences for the nonrotating case. The reason for this is that in the nonrotating case there is a global flow organization, which can be influenced by the aspect ratio. In the rotating regime vertically aligned vortices, in which most of the heat transport takes place [36,37], form the dominant feature of the flow. As this is a local effect, the heat transport in this regime does not depend on the aspect ratio. In the latter part of the paper we will analyze the vortex statistics for the different aspect ratios to support that the vortices are indeed a local phenomenon.

\section{EXPERIMENTAL PROCEDURE}

Various heat transport measurements on RRB convection were performed by Ahlers and co-workers in Santa Barbara. These measurements were done in a sample with an aspect ratio $\Gamma=1$ and cover the Ra number range $3 \times 10^{8} \lesssim \mathrm{Ra} \lesssim$ $2 \times 10^{10}$, the Pr number range $3.0 \lesssim \operatorname{Pr} \lesssim 6.4$, and the $1 /$ Ro number range $0 \lesssim 1 /$ Ro $\lesssim 20$. These data points are shown in the Ra-Ro- $\Gamma$ phase diagram for the RRB convection shown in Fig. 1. The experimental procedure that has been used in these experiments is described in detail by Zhong and Ahlers [17].
All the Nusselt number measurements of Zhong and Ahlers [17] were documented in the accompanying supplementary material of that paper (see Ref. [38]) and we use those data in our figures here. Recently, Weiss and Ahlers [21] did similar measurements in a sample with an aspect ratio $\Gamma=0.5$ and we find a very good agreement between their measurement results at $\mathrm{Ra}=4.52 \times 10^{9}$ and our simulation results, see Fig. 2(a).

Zhong and Ahlers [17] restricted themselves to aspect ratio $\Gamma=1$. Here we present heat transport measurements from the Eindhoven RB setup [18], which is based on the Santa Barbara design [17,39], for aspect ratio $\Gamma=4 / 3$ and 2.0. We also present one $\Gamma=1.0$ measurement for one particular relatively small Ra number from the Eindhoven setup because (i) this particular Ra number was not available in the Santa Barbara (SB) data set of Zhong and Ahlers [17] and we wanted to compare the results with those for $\Gamma=2$ and $4 / 3$ at the same $\mathrm{Ra}$, (ii) we want to compare with the simulation results that are restricted to small Ra, and (iii) we wanted to benchmark the results of our setup against those of the SB setup.

The Eindhoven RB convection sample has a diameter $D$ of $250 \mathrm{~mm}$ and due to the modular design of the setup measurements for different aspect ratios $(\Gamma=1.0-2.0)$ can be performed by replacing the sidewall. All measurements are performed with a mean temperature of $40^{\circ} \mathrm{C}(\mathrm{Pr}=4.38)$. For any given data point, measurements over typically the first $4 \mathrm{~h}$ were discarded to avoid transients and data taken over an additional period of at least another $8 \mathrm{~h}$ were averaged to get the average plate temperatures and the required power. Details about the setup and the experimental procedure, which is closely based on the Santa Barbara one [17,39], can be found in Ref. [18].

Presently, the Eindhoven experiments can only be performed with one type of plate material (copper) and not with more, as done in Santa Barbara. For this reason we can not apply the plate corrections to obtain the absolute Nusselt number without making some assumptions that cannot be verified at the moment. Therefore, for the experimental results we restrict ourself to relative Nusselt numbers $\mathrm{Nu}(1 / \mathrm{Ro}) / \mathrm{Nu}(0)$.

\section{NUMERICAL PROCEDURE}

In the simulations the flow characteristics of RRB convection for $\mathrm{Ra}=2.91 \times 10^{8}-4.52 \times 10^{9}, \operatorname{Pr}=4.38,0<$ $1 /$ Ro $<12.5$, and $0.5<\Gamma<2.0$ (see also Table I and Fig. 1) are obtained from solving the three-dimensional Navier-Stokes equations within the Boussinesq approximation:

$$
\begin{aligned}
& \frac{D \mathbf{u}}{D t}=-\nabla P+\left(\frac{\mathrm{Pr}}{\mathrm{Ra}}\right)^{1 / 2} \nabla^{2} \mathbf{u}+\theta \hat{\mathbf{z}}-\frac{1}{\mathrm{Ro}} \hat{\mathbf{z}} \times \mathbf{u}, \\
& \frac{D \theta}{D t}=\frac{1}{(\mathrm{PrRa})^{1 / 2}} \nabla^{2} \theta,
\end{aligned}
$$

with $\boldsymbol{\nabla} \cdot \mathbf{u}=0$. Here $\hat{\mathbf{z}}$ is the unit vector pointing in the direction opposite to gravity, $D / D t=\partial_{t}+\mathbf{u} \cdot \nabla$ is the material derivative, $\mathbf{u}$ is the velocity vector (with no-slip boundary conditions at all walls), and $\theta$ is the nondimensional temperature $0 \leqslant \theta \leqslant 1$. Finally, $P$ is the reduced pressure (separated from its hydrostatic contribution, but containing the centripetal contributions) $P=p-r^{2} / 8 \mathrm{Ro}^{2}$, with $r$ the 

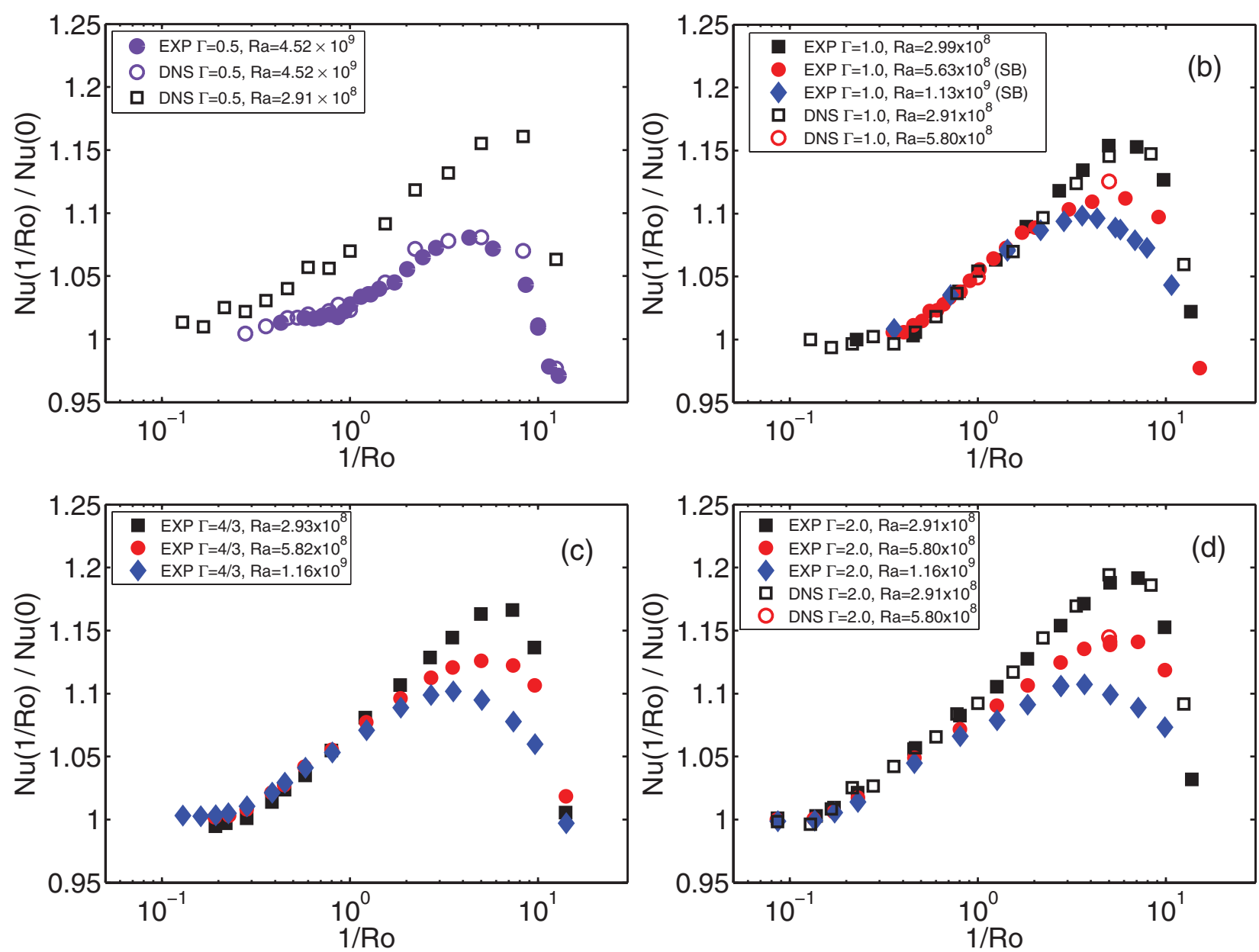

FIG. 2. (Color online) The ratio $\mathrm{Nu}(1 / \mathrm{Ro}) / \mathrm{Nu}(0)$ as a function of $1 / \operatorname{Ro}$ for different $\mathrm{Ra}$ for (a) $\Gamma=0.5$, (b) $\Gamma=1$, (c) $\Gamma=4 / 3$, and (d) $\Gamma=2$. (a) The experimental results for $\mathrm{Ra}=4.52 \times 10^{9}$ (run E13 of Weiss and Ahlers [21]) is indicated by the solid purple circles. The results from the DNS at $\mathrm{Ra}=2.91 \times 10^{8}$ and $\mathrm{Ra}=4.52 \times 10^{9}$ are indicated by the (black) open squares and (purple) open circles, respectively. (b) The experimental results for $\mathrm{Ra}=2.99 \times 10^{8}, 5.63 \times 10^{8}$ (run E4 of Zhong and Ahlers [17]), and 1.13 $\times 10^{9}$ (run E5 of Zhong and Ahlers [17]) are indicated by (black) solid squares, (red) solid circles, and (blue) solid diamonds, respectively. The DNS results for $\mathrm{Ra}=2.91 \times 10^{8}$ and $5.80 \times 10^{8}$ are indicated by black open squares and red open circles, respectively. (c) The experimental results for $\mathrm{Ra}=2.93 \times 10^{8}, 5.82 \times 10^{8}$, and $1.16 \times 10^{9}$ are indicated by (black) solid squares, (red) solid circles, and (blue) solid diamonds, respectively. (d) The experimental results for $\mathrm{Ra}=2.91 \times 10^{8}, 5.80 \times 10^{8}$, and $1.16 \times 10^{9}$ are indicated by (black) solid squares, (red) solid circles, and (blue) solid diamonds, respectively. The DNS results for Ra $=2.91 \times 10^{8}$ and $5.80 \times 10^{8}$ are indicated by (black) open squares and (red) open circles, respectively. All data presented in this figure are for $\operatorname{Pr}=4.38$.

distance to the rotation axis. The equations have been made nondimensional by using, next to $L$ and $\Delta$, the free-fall velocity $U=\sqrt{\beta g \Delta L}$. A constant temperature boundary condition is used at the bottom and top plate and the sidewall is adiabatic. Further details about the numerical procedure can be found in Refs. [40-42].

The resolutions used for the simulations are summarized in Table I. These grids allow for a very good resolution of the small scales both inside the bulk of turbulence and in the BLs where the grid-point density has been enhanced. We checked this by calculating the convergence of the volume-averaged kinetic $\epsilon_{u}$ and thermal $\epsilon_{\theta}$ dissipation rates as is proposed by Stevens et al. [43]. We find that these quantities always converge within a 5\% margin at most. A comparison with the results of Stevens et al. [43] shows that this convergence rate is clearly sufficient to reliably calculate the heat transfer. As argued by Shishkina et al. [44] it is especially important to properly resolve the BLs. According to Eq. (42) of that paper the minimal number of nodes that should

TABLE I. Simulations performed in this study. The columns from left to right indicate the following: the Rayleigh number Ra, the aspect ratio $\Gamma$, the inverse Rossby number $1 /$ Ro, the number of Ro cases that are simulated $\left(n_{\mathrm{Ro}}\right)$, and the number of grid points in the azimuthal, radial, and axial directions $\left(N_{\theta} \times N_{r} \times N_{z}\right)$.

\begin{tabular}{lcccc}
\hline \hline $\mathrm{Ra}$ & $\Gamma$ & $1 / \mathrm{Ro}$ & $n_{\mathrm{Ro}}$ & $N_{\theta} \times N_{r} \times N_{z}$ \\
\hline $2.91 \times 10^{8}$ & 0.5 & $0-12.5$ & 16 & $257 \times 97 \times 289$ \\
$4.52 \times 10^{9}$ & 0.5 & $0-12.5$ & 18 & $641 \times 161 \times 641$ \\
$2.91 \times 10^{8}$ & 1.0 & $0-12.5$ & 16 & $385 \times 193 \times 289$ \\
$5.80 \times 10^{8}$ & 1.0 & $0-5.0$ & 3 & $641 \times 193 \times 385$ \\
$2.91 \times 10^{8}$ & 2.0 & $0-12.5$ & 18 & $769 \times 385 \times 289$ \\
$5.80 \times 10^{8}$ & 2.0 & $0-5.0$ & 2 & $1281 \times 385 \times 385$ \\
\hline \hline
\end{tabular}


be placed in the thermal $\mathrm{BL}$ is $N_{\mathrm{BL}}=4.7\left(N_{\mathrm{BL}}=7.1\right)$ when $\mathrm{Ra}=2.91 \times 10^{8}\left(\mathrm{Ra}=4.52 \times 10^{9}\right)$ and $\mathrm{Pr}=4.38$. In the simulations at $\mathrm{Ra}=2.91 \times 10^{8}\left(\mathrm{Ra}=4.52 \times 10^{9}\right)$ we placed $N_{\mathrm{BL}}=14\left(N_{\mathrm{BL}}=22\right)$ points in the thermal BL, which is on the safe side. For the nonrotating case similar numbers are obtained for the grid-point resolution in the kinetic BL. When rotation is applied the kinetic BL becomes thinner. This effect only becomes significant for the highest 1/Ro number cases considered here and we emphasize that for all cases the number of points in the kinetic BL is above the criterion put forward by Shishkina et al. [44]. Furthermore, it is also very important to make sure that the results are statistically converged. Again, we use the methods introduced by Stevens et al. [43] to check this. For all cases the convergence is on the order of $1 \%$ and it is much better for most. For the simulations at $\mathrm{Ra}=2.91 \times 10^{8}$ in the aspect ratios $\Gamma=0.5,1.0$, and 2.0 the average statistical uncertainty is only about $0.5 \%$.

As a last check we compare the numerical results with experimental data. For this we use the data from Ahlers and coworkers, who did high precision heat transport measurements in $\Gamma=0.5$ [9], $\Gamma=1.0$ [45], and $\Gamma=2.0$ [45] samples. For all cases we find that the numerical results are within $1 \%$ of these experimental data. For some cases the numerical data of the present simulations are even slightly below the experimental data. This is reassuring with respect to numerical resolution issues, as normally the heat transport in an underresolved simulation is larger than the actual heat transport (see Ref. [43]).

In the simulations we partially neglect centrifugal forces, namely, the density dependence of the centripetal forces, which in the Boussinesq equations show up as $-2 \operatorname{Fr} r \theta \hat{\mathbf{r}}$, with the radial unit vector $\hat{\mathbf{r}}$ [46]. Several authors [13,20,47] have shown that this is justified for small Froude numbers. For all experiments in the $\Gamma=1$ sample, where the Froude number $\mathrm{Fr}=\Omega^{2}(L / 2) / g$ is below 0.03 for all cases, this condition is fulfilled. For the experiments in the $\Gamma=2$ sample the Froude number is below 0.05 for all experiments up to $1 /$ Ro $=5$. For higher 1/Ro the Froude number quickly increases up to 0.12 for $\mathrm{Ra}=2.91 \times 10^{8}$ and up to 0.49 for $\mathrm{Ra}=1.16 \times 10^{9}$. For the experiments at $\mathrm{Ra}=2.91 \times 10^{8}$, for which the highest Froude number is 0.12 , this does not influence the results as we find perfect agreement between the experimental and numerical results [see Fig. 2(c)]. The experimental results for higher $\mathrm{Ra}$ in the $\Gamma=2$ sample, where the Froude numbers are higher for the highest $1 /$ Ro case, do not suggest a strong influence of this effect on the heat transport measurements. However, at the moment we cannot completely rule it out either.

We note that the simulations presented here are very CPU time intensive (about $1.5 \times 10^{6}$ standard DEISA CPU hours have been used) due to the large aspect ratios, the relatively high $\mathrm{Ra}$ numbers that are resolved with high resolution, the number of different Ro number cases, and the long averaging times that are needed to get sufficient statistical convergence.

\section{RESULTS}

In Fig. 2 the heat transport enhancement with respect to the nonrotating case is shown for $\Gamma=0.5,1,4 / 3$, and 2 and different Ra. Here we find excellent agreement between experimental and numerical results. The figure shows that there is a strong heat transport enhancement due to Ekman pumping

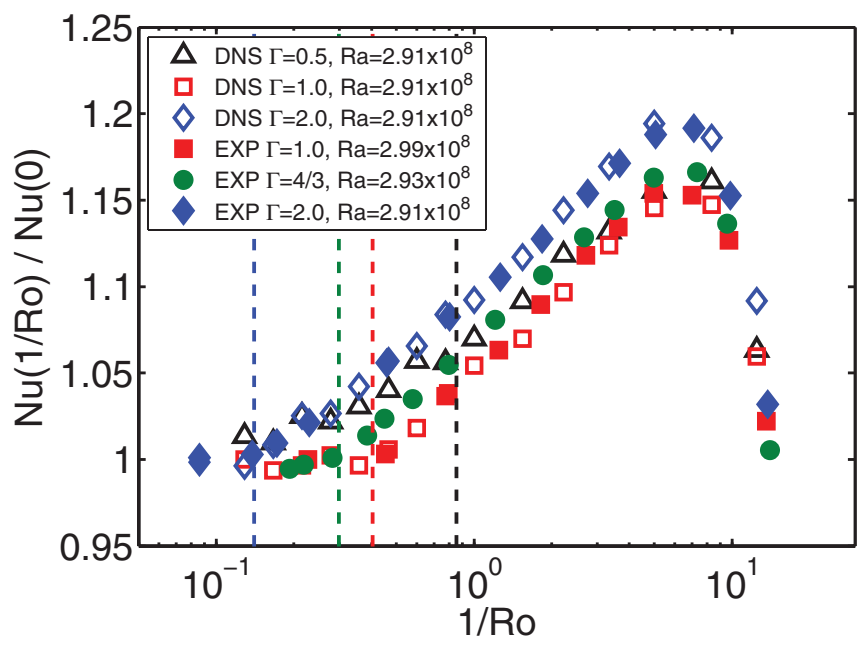

FIG. 3. (Color online) The ratio $\mathrm{Nu}(1 / \mathrm{Ro}) / \mathrm{Nu}(0)$ as a function of $1 /$ Ro for $\mathrm{Ra} \approx 3 \times 10^{8}$ and different $\Gamma$. The experimental results for $\Gamma=1,4 / 3$, and 2 are indicated by (red) solid squares, (green) solid circles, and (blue) solid diamonds, respectively. The DNS results for $\Gamma=0.5,1$, and 2 are indicated by (black) open triangles, (red) open squares, and (blue) open diamonds, respectively. All data presented are for $\operatorname{Pr}=4.38$.

when $1 / \operatorname{Ro}>1 / \operatorname{Ro}_{c}$, where $1 / \operatorname{Ro}_{c}$ indicates the position of the onset of heat transport enhancement $[14,15]$. The figure also shows that the heat transport enhancement decreases with increasing $\mathrm{Ra}$. This is because the eddy thermal diffusivity is larger at higher Ra and this causes the warm (cold) fluid that enters the base of the vortices to spread out more quickly in the middle of the sample. This makes the effect of Ekman pumping smaller and this results in a lower heat transport enhancement. The breakdown of $\mathrm{Nu}$ at high $1 / \mathrm{Ro}$ is an effect

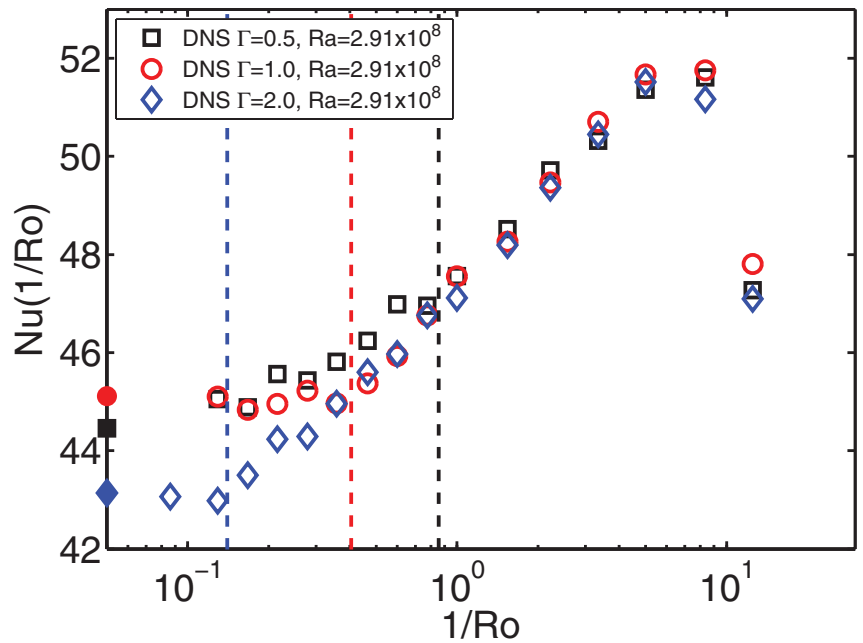

FIG. 4. (Color online) Absolute Nusselt number of the simulation data presented in Fig. 3. The nonrotating values are indicated by the solid symbols on the left-hand side. The results for $\Gamma=0.5,1$, and 2 are indicated by (black) open squares, (red) open circles, and (blue) open diamonds, respectively. From right to left, the (black, red, and blue) dashed vertical lines indicate $1 / \operatorname{Ro}_{c}$ for $\Gamma=0.5,1$, and 2 , respectively. All data presented are for $\operatorname{Pr}=4.38$. 

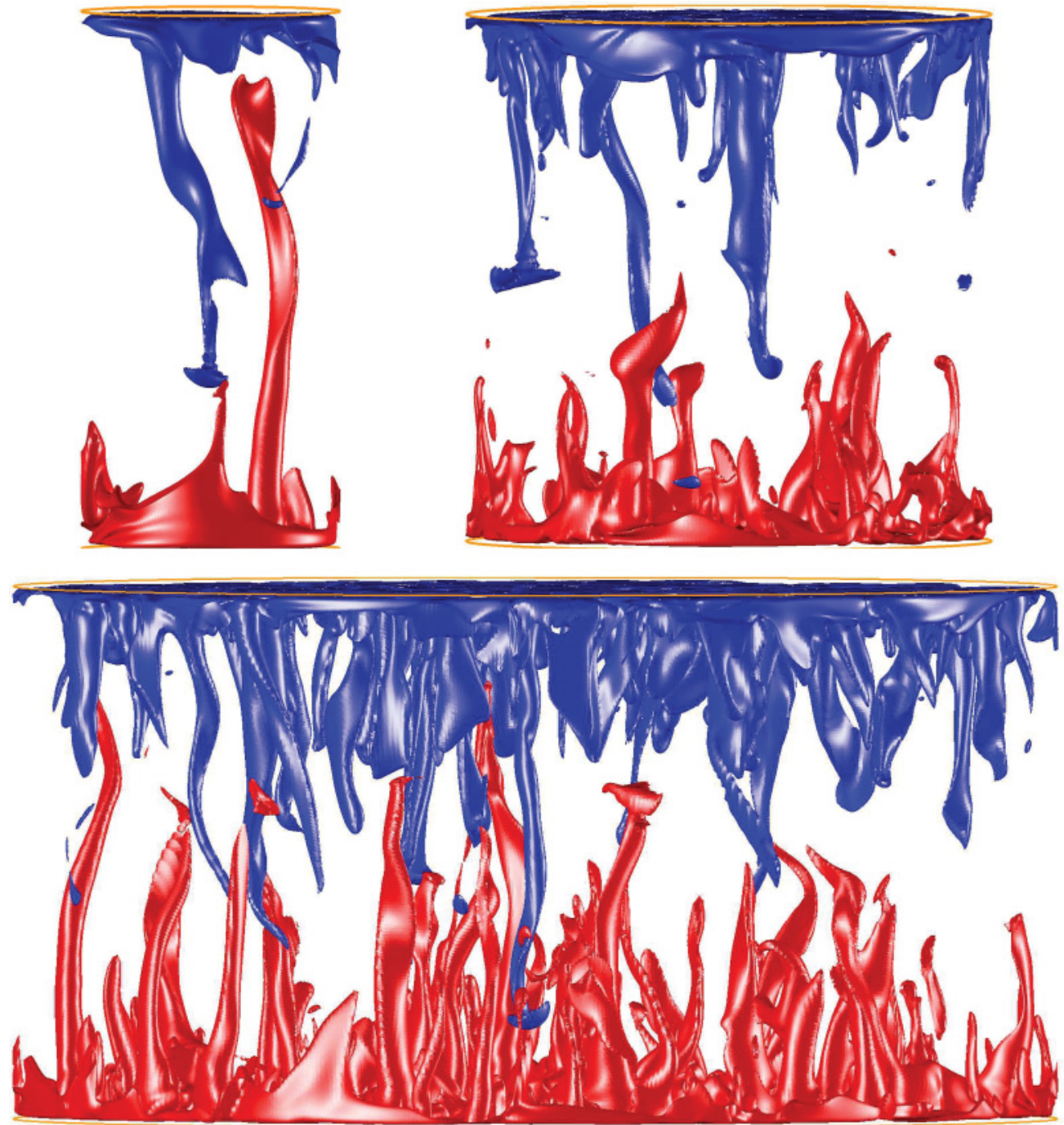

FIG. 5. (Color online) Three-dimensional visualization of the temperature isosurfaces in the cylindrical sample at $0.65 \Delta$ [gray (red, originating from the bottom)] and $0.35 \Delta$ [black (blue, originating from the top)], respectively, for $\operatorname{Ra}=2.91 \times 10^{8}, \operatorname{Pr}=4.38,1 / \operatorname{Ro}=3.33$, and $\Gamma=0.5$ (left top plot), $\Gamma=1.0$ (right top plot), and $\Gamma=2.0$ (bottom plot).

of the suppression of vertical velocity fluctuations through the strong rotation.

When the results for $\Gamma=0.5,1,4 / 3$, and 2 are compared, they look pretty similar at first sight, i.e., there is a strong heat transport enhancement when $1 / \mathrm{Ro}>1 / \mathrm{Ro}_{c}$ and a heat transport reduction for very strong rotation rates. However, when we look more closely we see that there are several important differences. First of all, $1 / \mathrm{Ro}_{c}$, the rotation rate needed to get heat transport enhancement, increases with decreasing aspect ratio. Weiss et al. $[15,21]$ already observed this and explained it by finite-size effects. In addition, a close comparison between the $\Gamma=1$ and 2 data in Fig. 2 shows that the heat transport enhancement is slightly larger in a $\Gamma=2$ sample than in a $\Gamma=1$ sample. This can be observed in more detail in Fig. 3, where the relative heat transport enhancement at fixed $\mathrm{Ra}$ is compared for several aspect ratios.

The absolute heat transfer shown in Fig. 4 is available only for the numerical case (as explained in Sec. II). In that figure we see that there are some visible differences in the heat transport for the nonrotating case, i.e., without rotation we find that the heat transport in a $\Gamma=2.0$ sample is approximately $5 \%$ lower than in a $\Gamma=1.0$ sample. Similar differences in the heat transport as a function of $\Gamma$ have been shown by the numerical study of Bailon-Cuba et al. [48] and in experiments of Funfschilling et al. [45] and Sun et al. [49], although these experimental and numerical results seem to suggest that the heat transport becomes less dependent on the aspect ratio for higher Ra. The main point indicated by Fig. 4 is that the heat transport becomes independent of the aspect ratio once $1 / \mathrm{Ro} \gg 1 / \mathrm{Ro}_{c}$, with $1 / \mathrm{Ro}_{c} \approx 0.14,0.4$, and 0.86 for $\Gamma=2,1$, and 0.5 , respectively. We note that we also find for $\mathrm{Ra}=5.80 \times 10^{8}$ that the difference in Nusselt number between the cases for $\Gamma=1$ and 2 is smaller for $1 / \operatorname{Ro}=5$ than for the nonrotating case, which is in agreement with the data presented in Fig. 4.

We believe that the reason for this phenomenon lies in the flow structures that are formed. For the nonrotating case the flow organizes globally in the large-scale convection roll. Because this global flow structure can depend on the 

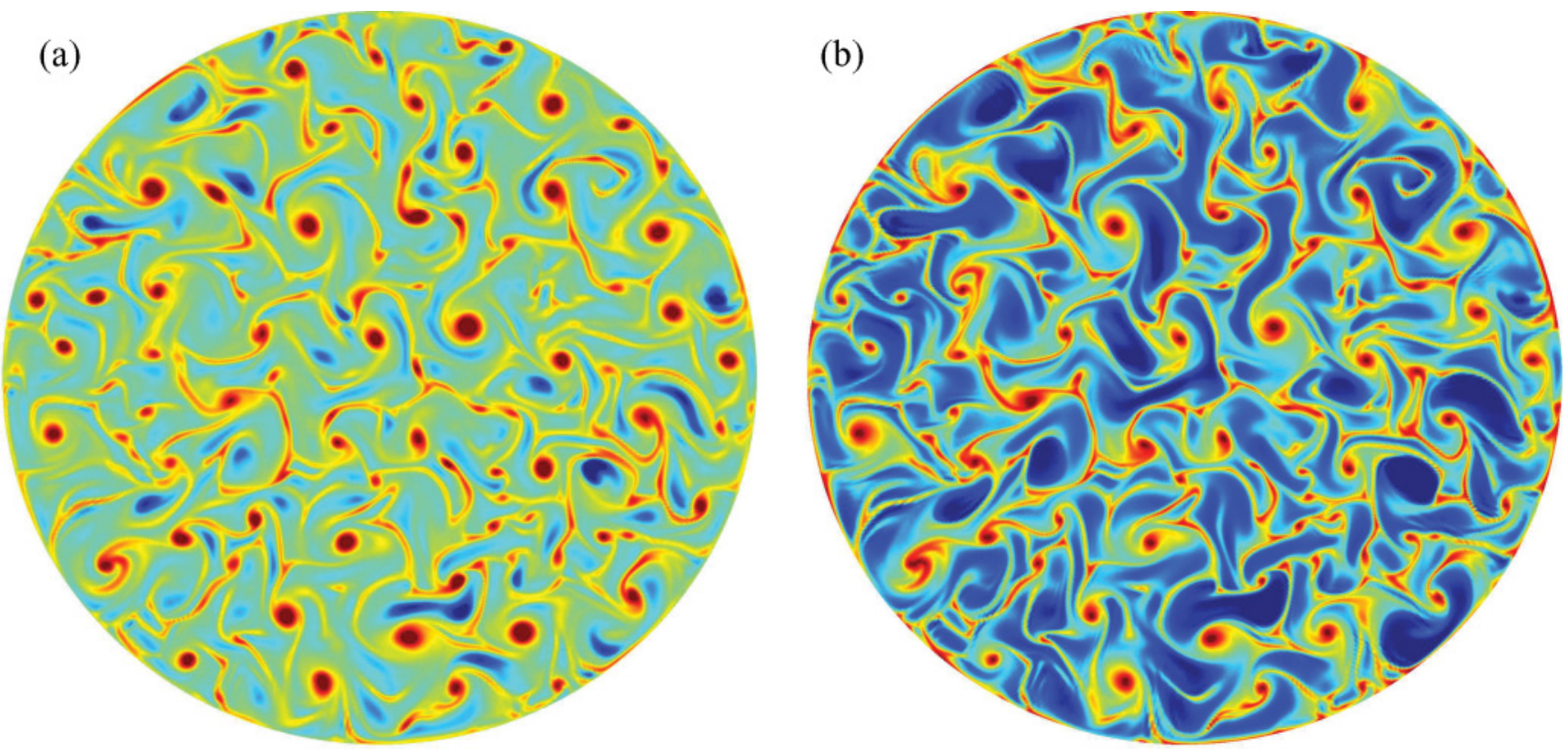

FIG. 6. (Color online) Visualization of the vertical velocity (left plot) and temperature (right plot) fields at the kinetic BL height for $\mathrm{Ra}=2.91 \times 10^{8}, \operatorname{Pr}=4.38,1 / \mathrm{Ro}=5$, and $\Gamma=2$. Note that there is a strong correlation between the areas where the strongest vertical velocity and highest temperatures are found, namely, in the vortices. Gray (red) and black (blue) indicate (a) upflowing (warm) and (b) downflowing (cold) fluid.

aspect ratio, there can be small variations in the Nusselt number as a function of the aspect ratio. For strong enough rotation, i.e., $1 / \mathrm{Ro} \gg 1 / \mathrm{Ro}_{c}$, the global LSC is replaced by vertically aligned vortices as the dominant feature of the flow $[13,14,16,17,22,47]$. In this regime most of the heat transport takes place in vertically aligned vortices [36,37,50,51]. Because the vortices are a local effect, the influence of the aspect ratio on the heat transport in the system should be negligible.
This assumption is used in several models [36,37,52], which consider a horizontally periodic domain, that are developed to understand the heat transport in rotating turbulent convection.

To investigate this idea we made three-dimensional visualizations of the temperature isosurfaces at $\mathrm{Ra}=2.91 \times 10^{8}$, $\operatorname{Pr}=4.38$, and $1 / \mathrm{Ro}=3.33$ for the different aspect ratios (see Fig. 5). Indeed, the figures confirm that vertically aligned vortices are formed in all aspect-ratio samples. Furthermore,
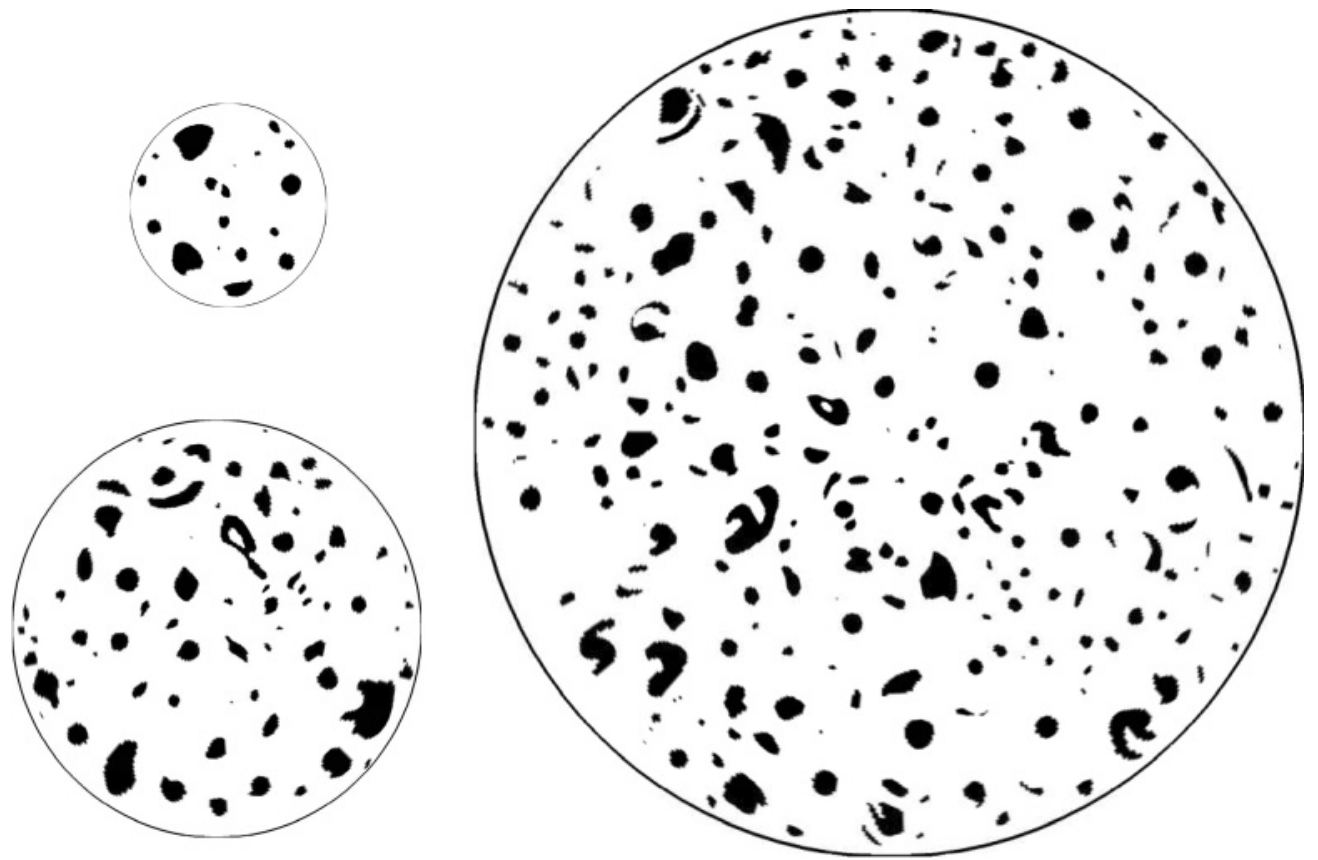

FIG. 7. Vortices at the edge of the kinetic BL as identified by the $Q_{2 \mathrm{D}}$ criterion for $\mathrm{Ra}=2.91 \times 10^{8}, \operatorname{Pr}=4.38,1 / \mathrm{Ro}=5$, and $\Gamma=0.5$ (top left plot), $\Gamma=1.0$ (bottom left plot), and $\Gamma=2.0$ (right plot). 


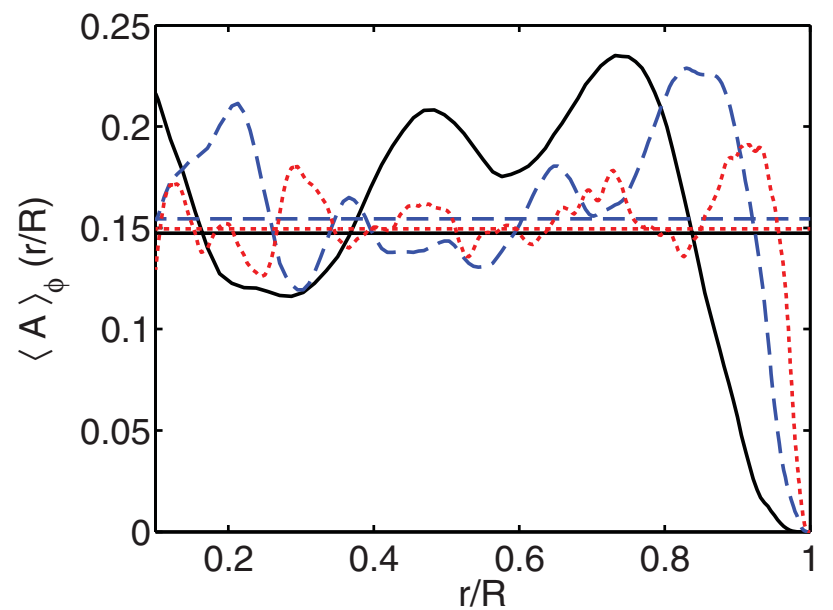

(a)

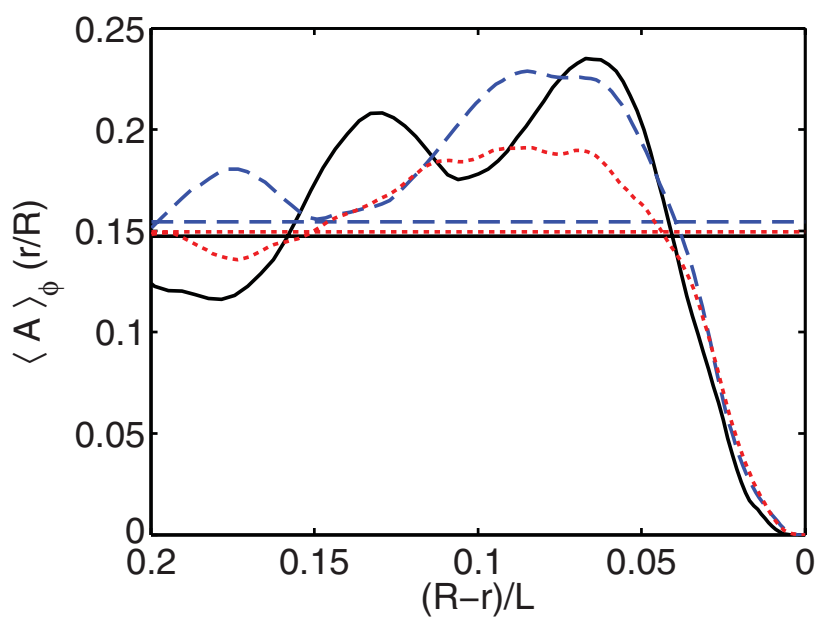

(b)

FIG. 8. (Color online) Straight lines indicate the average of the fraction of the horizontal area $A$ covered by vortices for Ra $=2.91 \times 10^{8}$, $\operatorname{Pr}=4.38,5<1 /$ Ro $<8.33$, and different aspect ratios. The corresponding (black) solid line, (blue) dashed line, and (red) dotted line indicate the azimuthal average of the fraction of the area that is covered by vortices as a function of the radial position, i.e. $\langle A\rangle_{r / R}$, for $\Gamma=0.5,1.0$, and 2.0, respectively. The data are shown as a function of (a) $r / R$ and (b) $(R-r) / L$ close to the sidewall. For each aspect ratio the data are averaged for the simulation data at $1 /$ Ro $=5$ and 8.33 . For each rotation rate two snapshots at the bottom and top BLs are used.

the figure reveals that a larger number of vortices is formed in the $\Gamma=1$ and 2 samples than in the $\Gamma=0.5$ sample. This is expected since these samples have a larger horizontal extension.

In order to reveal the structure of the flow in more detail we show the temperature and vertical velocity fields at the kinetic BL height near the bottom plate for $\mathrm{Ra}=2.91 \times 10^{8}$, $\operatorname{Pr}=4.38,1 / \operatorname{Ro}=5$, and $\Gamma=2$ in Fig. 6 . This figure clearly shows that hot fluid is captured in the up-going vortices. Similar plots (not shown) revealed that cold fluid near the top plate is captured in down-going vortices. In order to study the vortex statistics one needs to have a clear criterion of what exactly constitutes a vortex. For this we use the so-called $Q$ criterion $[15,34,50,53,54]$. This criterion requires that the quantity $Q_{2 \mathrm{D}}[14,55]$, which is a quadratic form of various velocity gradients, is calculated in a plane of fixed height. Here we always take the kinetic BL height. Following Weiss et al. [15], an area is identified as a vortex when $Q_{2 \mathrm{D}}<-\left\langle\left|Q_{2 \mathrm{D}}\right|\right\rangle_{v}$, where $\left\langle\left|Q_{2 \mathrm{D}}\right|\right\rangle_{v}$ is the volume-averaged value of the absolute values of $Q_{2 \mathrm{D}}$. Here we have set the threshold for the vortex detection more restrictive than in Ref. [15] to make sure that only the strong up-going (down-going) vortices are detected. However, we note that similar results are obtained when a less restrictive threshold is used.

The result of this procedure for $\mathrm{Ra}=2.91 \times 10^{8}, 1 / \mathrm{Ro}=$ 5 , and $\Gamma=0.5-2.0$ is shown in Fig. 7. The figure shows that the vortices (both up- and down-going) are, in general, randomly distributed. However, note that no vortices are formed close to the sidewall. To quantify this we determined the radial distribution of the vortices from the plots shown in Fig. 7 and similar plots. The result is shown in Fig. 8. Figure 8(a) confirms that no vortices are formed close to the sidewall, while in the bulk their fraction is roughly constant. Figure 8(b) shows that the size of the region close to the sidewall where no vortices are formed is roughly independent of the aspect ratio. This is in agreement with the predication of Weiss and Ahlers [21] that is derived from a phenomenological Ginzburg-Landau model. As detailed information about the flow field is needed to determine the vortex distribution, it is very hard to obtain these data from experimental measurements [54]. In Fig. 8(b) one can see that in the region $(R-r) / L \lesssim 0.015$ the value of $\langle A\rangle_{r / R}$ decreases faster to zero than for $(R-r) / L \gtrsim 0.015$, which is due to the vortex detection method employed in this study. More specifically, we detect only the core of the vortex. As the vortex core is always formed some distance away from the wall this causes an (artificial) enhanced decrease in the number of vortices that are detected in the direct vicinity of the wall. Due to the vortex detection method that is used it is also difficult to estimate the average radius of the vortices as only the core of the vortex is detected.

However, the main point here is that, as one can see in Fig. 8, the fraction of the horizontal area that is covered by vortices (see the horizontal lines in the figure) is independent of the aspect ratio. This observation supports our finding that the heat transport is independent of the aspect ratio in the rotating regime. This result may seem somewhat unexpected based on the data shown in Fig. 8(b). This figure specifically shows that the absolute size of the vortex-depleted region close to the sidewall is approximately aspect-ratio independent. Therefore, one would have expected that the average horizontal area that is covered by vortices averaged over the whole area is higher for larger aspect ratio because for larger-aspect-ratio samples this vortex-depleted sidewall region is smaller than for smaller-aspect-ratio samples. However, just next to the vortex-depleted region we find the vortex-enhanced region. As is shown in Fig. 8(b), the absolute width of this region seems to be rather independent of the aspect ratio of the sample too. Hence the effects of the vortex-depletion and vortex-enhancement regions on the horizontally averaged area that is covered by vortices cancel out in first order.

In this paper we focused on the influence of the aspect ratio on the fraction of the horizontal area that is covered 
with vortices. We note that Weiss et al. [15] have already investigated the effect of rotation rate on the fraction of the horizontal area that is covered by vortices. There we showed that the average horizontal area that is covered by vortices increases approximately linearly with $1 /$ Ro when $1 / \mathrm{Ro}>1 / \mathrm{Ro}_{c}$. This result is in agreement with the predictions obtained from a phenomenological Ginzburg-Landau-like model that is discussed in that paper. Furthermore, it was shown by Kunnen et al. [54] that the vortex densities and mean vortex radii are mostly independent of the Taylor number $\mathrm{Ta}=\mathrm{Ra} /\left(\mathrm{Ro}^{2} \mathrm{Pr}\right)$ except very close to the bottom and top plates where more vortices are detected when the Taylor number is raised. As the numerical simulations considered here have been obtained for similar $\mathrm{Ra}$ and $\mathrm{Pr}$ as the ones used in the above studies, we have not investigated the influence of these parameters on the vortex statistics.

\section{CONCLUSION}

In summary, we investigate the effect of the aspect ratio on the heat transport in turbulent rotating Rayleigh-Bénard convection by results obtained from experiments and direct numerical simulations. We find that the heat transport in the rotating regime is independent of the aspect ratio, although there are some visible differences in the heat transport for the different aspect ratios in the nonrotating regime at $\mathrm{Ra}=$ $2.91 \times 10^{8}$. This is because in the nonrotating regime the aspect ratio can influence the global flow structure. However, in the rotating regime most heat transport takes place in vertically aligned vortices, which are a local effect. Based on the simulation results, we find that the fraction of the horizontal area that is covered by the vortices is independent of the aspect ratio, which confirms that the vertically aligned vortices are indeed a local effect. This supports the simulation results, which show that the heat transport becomes independent of the aspect ratio in the rotating regime. In addition, it confirms the main assumption that is used in most models, which consider a horizontally periodic domain [36,37,52], that are developed to understand the heat transport in rotating turbulent convection. The analysis of the vortex statistics also revealed that the vortex concentration is reduced close to the sidewall, while the distribution is nearly uniform in the center. In between these two regions, there is a region of enhanced vortex concentration. The widths of both that region and the vortex-depleted region close to the sidewall are independent of the aspect ratio. This analysis highlights the value of numerical simulations in turbulence research: The determination of the vortex distribution requires detailed knowledge of the flow field and therefore it would have been very difficult to obtain this finding purely from experimental measurements.

\section{ACKNOWLEDGMENTS}

We gratefully acknowledge various discussions with Guenter Ahlers over this line of research and his helpful comments on our manuscript. The authors wish to thank Eric de Cocq, Gerald Oerlemans, and Freek van Uittert (design and manufacturing of the experimental setup) for their contributions to this work and Jaap van Wensveen of Tempcontrol for advice and helping to calibrate the thermistors. We thank Chao Sun for stimulating discussions. We thank the DEISA Consortium (www.deisa.eu), co-funded through the EU FP7 Project No. RI-222919, for support within the DEISA Extreme Computing Initiative. We thank Wim Rijks (SARA) and Siew Hoon Leong (Cerlane) (LRZ) for support during the DEISA project. The simulations were performed on the Huygens cluster (SARA) and HLRB-II cluster (LRZ). R.J.A.M.S. was financially supported by the Foundation for Fundamental Research on Matter.
[1] G. Ahlers, S. Grossmann, and D. Lohse, Rev. Mod. Phys. 81, 503 (2009).

[2] D. Lohse and K. Q. Xia, Annu. Rev. Fluid Mech. 42, 335 (2010).

[3] S. Grossmann and D. Lohse, J. Fluid. Mech. 407, 27 (2000).

[4] S. Grossmann and D. Lohse, Phys. Rev. Lett. 86, 3316 (2001).

[5] S. Grossmann and D. Lohse, Phys. Rev. E 66, 016305 (2002).

[6] S. Grossmann and D. Lohse, Phys. Fluids 16, 4462 (2004).

[7] C. Sun, H. D. Xi, and K. Q. Xia, Phys. Rev. Lett. 95, 074502 (2005).

[8] H. D. Xi and K. Q. Xia, Phys. Fluids 20, 055104 (2008).

[9] S. Weiss and G. Ahlers, J. Fluid. Mech. 676, 5 (2011).

[10] E. van der Poel, R. Stevens, and D. Lohse, Phys. Rev. E 84, 045303(R) (2011).

[11] K.-Q. Xia, J. Fluid. Mech. 676, 1 (2011).

[12] B. M. Boubnov and G. S. Golitsyn, J. Fluid Mech. 219, 215 (1990).

[13] J.-Q. Zhong, R. J. A. M. Stevens, H. J. H. Clercx, R. Verzicco, D. Lohse, and G. Ahlers, Phys. Rev. Lett. 102, 044502 (2009).

[14] R. J. A. M. Stevens, J.-Q. Zhong, H. J. H. Clercx, G. Ahlers, and D. Lohse, Phys. Rev. Lett. 103, 024503 (2009).
[15] S. Weiss, R. Stevens, J.-Q. Zhong, H. Clercx, D. Lohse, and G. Ahlers, Phys. Rev. Lett. 105, 224501 (2010).

[16] R. P. J. Kunnen, B. J. Geurts, and H. J. H. Clercx, J. Fluid Mech. 82, 445 (2010).

[17] J.-Q. Zhong and G. Ahlers, J. Fluid Mech. 665, 300 (2010).

[18] R. P. J. Kunnen, R. J. A. M. Stevens, J. Overkamp, C. Sun, G. J. F. van Heijst, and H. J. H. Clercx, J. Fluid Mech. (2011), doi: $10.1017 / \mathrm{jfm} .2011 .383$.

[19] J. E. Hart, S. Kittelman, and D. R. Ohlsen, Phys. Fluids 14, 955 (2002).

[20] R. P. J. Kunnen, H. J. H. Clercx, and B. J. Geurts, Europhys. Lett. 84, 24001 (2008).

[21] S. Weiss and G. Ahlers, J. Fluid. Mech. 684, 407 (2011).

[22] R. J. A. M. Stevens, H. J. H. Clercx, and D. Lohse, New J. Phys. 12, 075005 (2010).

[23] H. T. Rossby, J. Fluid Mech. 36, 309 (1969).

[24] Y. Liu and R. E. Ecke, Phys. Rev. Lett. 79, 2257 (1997).

[25] Y. Liu and R. E. Ecke, Phys. Rev. E 80, 036314 (2009).

[26] E. M. King, S. Stellmach, J. Noir, U. Hansen, and J. M. Aurnou, Nature (London) 457, 301 (2009). 
[27] K. Julien, S. Legg, J. McWilliams, and J. Werne, J. Fluid Mech. 322, 243 (1996).

[28] M. Sprague, K. Julien, E. Knobloch, and J. Werne, J. Fluid Mech. 551, 141 (2006).

[29] P. Oresta, G. Stingano, and R. Verzicco, Eur. J. Mech. 26, 1 (2007).

[30] S. Schmitz and A. Tilgner, Phys. Rev. E 80, 015305 (2009).

[31] S. Schmitz and A. Tilgner, Geophys. Astrophys. Fluid Dyn. 104, 481 (2010).

[32] R. J. A. M. Stevens, H. J. H. Clercx, and D. Lohse, Phys. Fluids 22, 085103 (2010).

[33] F. Zhong, R. E. Ecke, and V. Steinberg, J. Fluid Mech. 249, 135 (1993).

[34] P. Vorobieff and R. E. Ecke, J. Fluid Mech. 458, 191 (2002).

[35] J. Niemela, S. Babuin, and K. Sreenivasan, J. Fluid Mech. 649, 509 (2010).

[36] J. W. Portegies, R. P. J. Kunnen, G. J. F. van Heijst, and J. Molenaar, Phys. Fluids 20, 066602 (2008).

[37] I. Grooms, K. Julien, J. Weiss, and E. Knobloch, Phys. Rev. Lett. 104, 224501 (2010).

[38] See Supplemental Material at http://link.aps.org/supplemental/ 10.1103/PhysRevE.84.056313 for a movie showing the threedimensional phase diagram.

[39] E. Brown, D. Funfschilling, A. Nikolaenko, and G. Ahlers, Phys. Fluids 17, 075108 (2005).

[40] R. Verzicco and P. Orlandi, J. Comput. Phys. 123, 402 (1996).
[41] R. Verzicco and R. Camussi, J. Fluid Mech. 383, 55 (1999).

[42] R. Verzicco and R. Camussi, J. Fluid Mech. 477, 19 (2003).

[43] R. J. A. M. Stevens, R. Verzicco, and D. Lohse, J. Fluid. Mech. 643, 495 (2010).

[44] O. Shishkina, R. J. A. M. Stevens, S. Grossmann, and D. Lohse, New J. Phys. 12, 075022 (2010).

[45] D. Funfschilling, E. Brown, A. Nikolaenko, and G. Ahlers, J. Fluid Mech. 536, 145 (2005).

[46] G. M. Homsy and J. L. Hudson, J. Fluid Mech. 35, 33 (1969).

[47] R. P. J. Kunnen, H. J. H. Clercx, and B. J. Geurts, Phys. Rev. E 74, 056306 (2006).

[48] J. Bailon-Cuba, M. Emran, and J. Schumacher, J. Fluid Mech. 655, 152 (2010).

[49] C. Sun, K. Q. Xia, and P. Tong, Phys. Rev. E 72, 026302 (2005).

[50] B. M. Boubnov and G. S. Golitsyn, J. Fluid Mech. 167, 503 (1986).

[51] S. Sakai, J. Fluid Mech. 333, 85 (1997).

[52] S. Legg, K. Julien, J. McWilliams, and J. Werne, Phys. Chem. Earth, Part B: Hydrology, Oceans and Atmosphere 26, 259 (2001).

[53] P. Vorobieff and R. E. Ecke, Physica D 123, 153 (1998).

[54] R. P. J. Kunnen, B. J. Geurts, and H. J. H. Clercx, Phys. Rev. E 82, 036306 (2010).

[55] J. C. McWilliams, J. Fluid Mech. 146, 21 (1984). 